\title{
Mesoscale inertial transition in granular materials
}

\author{
Adriane Clerc $^{1 *}$, Antoine Wautier ${ }^{1}$, Stéphane Bonelli ${ }^{1}$, and François Nicot $^{2}$ \\ ${ }^{1}$ INRAE, Aix Marseille Univ., RECOVER, 3275 Rte Cézanne, CS 40061, 13182 Aix en Provence Cedex5, France \\ ${ }^{2}$ INRAE, Grenoble Alpes Univ., ETNA, 2 Rue de la Papeterie BP76, 38402 Saint Martin d'Hères, France
}

\begin{abstract}
Granular assemblies can experience complex failure patterns along a given loading path, with a distribution of ephemeral inertial events marked by local outbursts in kinetic energy. However, investigating such mechanisms appears to be necessary to understand how a certain failure mode develops in a granular material. Using a discrete element method, this study highlights several microstructure reorganizations before the specimen reaches a proper failure state. Meso structures have proven to be efficient to understand the elementary mechanisms responsible for these outbursts in kinetic energy. Strain-like and stress-like quantities are thus defined at a mesoscale and they are used to characterize the nucleation and propagation of these local microstructural events.
\end{abstract}

\section{Introduction}

Granular materials are still fascinating by their complex behaviour. One of the difficulties lies in the description of a media that behave either like a solid or a fluid. The transition between quasi static to inertial regime is still an active research topic [1,2]. Bursts of kinetic energy are seen as early evidence of mechanical instabilities $[3,4]$, and their analysis is consistent with the search for precursors of regime changes $[5,6]$. Another main challenge when dealing with granular materials is that they can be considered as a continuous media macroscopically but their fundamental components are discrete. As a consequence, inertial transitions and mechanical instabilities are visible at the macro scale, but their nucleation results from micro scale mechanisms. Thus, multi-scale approaches have been developed in order to bridge the gap between the two scales. In this study, grain-loops [7, 8] are used as an efficient way of tesselate a 2D sample domain and give relevant information on forces, strains and geometrical reorganizations.

This work is based on discrete element method simulations from YADE [9] in which grains are idealized by spheres interacting through linear elastofrictional laws [10]. Numerical experiments consist in the creation of an idealized quasi-2 $\mathrm{D}^{\mathrm{a}}$ dense sample of 25,000 grains subjected to a biaxial test. The biaxial test is composed of two phases. First, an isotropic compression of intensity $\sigma_{0}$ is applied. Secondly, a strain-controlled phase where the confining pressure $\sigma_{0}$ is kept constant on lateral boundaries while horizontal walls are moved with a strain rate $\dot{\varepsilon}$. The characteristics

\footnotetext{
*adriane.clerc@inrae.fr

${ }^{\text {a }}$ Note that while the simulations are 2D, the mass and inertia of the grains corresponds to the one of spheres. Indeed, a
}

of the sample and the loading are summarized in Table 1. DEM simulations give the expected response for deviatoric stress and volumetric strain corresponding to a dense granular material. For the sake of brevity the reader is referred to Clerc et al. (2021) [11] for more details.

\begin{tabular}{|c|c|}
\hline Parameters & Dense sample \\
\hline Grain density $\left(\mathrm{kg} / \mathrm{m}^{3}\right)$ & 3000 \\
\hline Friction coefficient & 0.7 \\
\hline$k_{n} / D(\mathrm{MPa})$ & 356 \\
\hline$k_{f} / k_{n}$ & 0.42 \\
\hline Grain size distribution & $\begin{array}{c}\text { Uniform with } \\
D_{\max } / D_{\min }=3.5\end{array}$ \\
\hline Loading rate $\dot{\varepsilon}\left(\mathrm{s}^{-1}\right)$ & 0.01 \\
\hline Confining pressure $\sigma_{0}(\mathrm{kPa})$ & 100 \\
\hline
\end{tabular}

Table 1. Dense sample parameters for the triaxial test. $D$ is the mean diameter of the grains.

\section{Focus on the bursts of kinetic energy}

Kinetic energy is attached to the velocities of the grain and it is thus a microscopic quantity. However, it can be integrated and defined at a larger scale (macro and meso scales). For spherical grains, the kinetic energy is:

$$
E_{c}^{g}=\frac{1}{2} m_{g}\left\|c_{g}\right\|^{2}+\frac{1}{5} m_{g}\left|w_{g} R_{g}\right|^{2}
$$

where $m_{g}$ is the grain mass, $R_{g}$ the grain radius, $c_{g}$ and $w_{g}$ are the translational and rotational velocity of the grain.

planar sample composed of one layer of spheres is considered. In order to use stresses, an out-of plane dimension equal to $D_{\max }$ is used. 
In order to detect a burst of kinetic energy, a threshold is defined based on the grain mean kinetic energy at the critical state (state where the sample deforms continuously with constant stress state and constant volume). From all the bursts that can be detected, we select four. For the sake of brevity, the results of a single burst are presented as the bursts present all the same patterns. The area of the burst propagation is separated from the rest of the sample domain to detect some microstructure features specific to the nucleation of the burst. It is easily done by setting an approximate control box around the location of the beginning of the burst (Fig. 1).

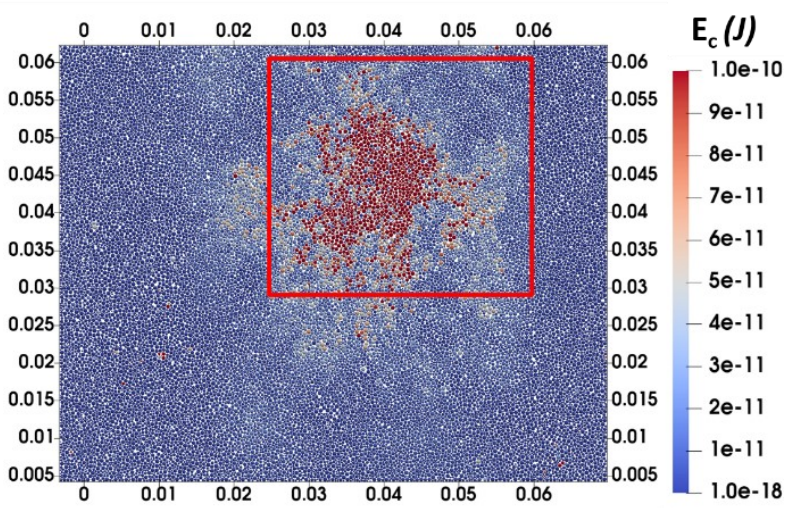

Fig. 1. Screenshot of the presented burst. The particles are colored in function of their kinetic energy. The box used for the delimitation of the burst is superimposed to the screenshot. It takes into account the slight displacement of the burst on the right direction (displacement not shown here).

Once the box is defined, preliminary investigations can be carried out. The sliding index $I_{p}$ for each contact is defined as the ratio between the norm of the tangential force and the norm of the normal force multiplied by the friction coefficient. This index is an indicator of the stability of a contact. The extreme values it can take are 0 (no tangential force) and 1 (contact sliding). The comparison of the probability density function of $I_{p}$ for contacts inside and outside the burst area reveals that the contacts inside the area are closer to their sliding limit just before the burst, while the probability density function outside the area does not change much during the burst. A sliding index close to 1 has already been shown to be a necessary condition to observe a burst of kinetic energy [4].

If we compare the different energies variations in the whole sample, we see that the kinetic energy goes through a peak corresponding. Meanwhile, the elastic energy decreases and the cumulative energy dissipated in plastic slips increases (Fig. 2). We can conclude that an excess of elastic energy stored into the contacts is transformed into kinetic energy but mostly dissipated through friction.

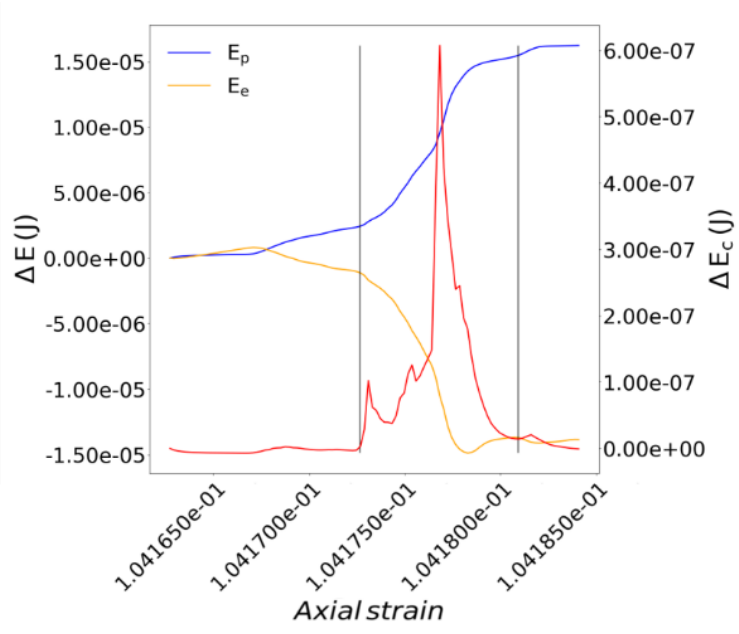

Fig. 2. Evolution of the energy dissipated through contact sliding $E_{p}$, of the elastic energy $E_{e}$ (left y-axis), and of the kinetic energy $E_{c}$ (in red, right y-axis) during the burst of kinetic energy presented (the duration of the burst is materialized by the black vertical lines). Only variations of energies are plotted, the reference energy levels being taken before the burst for an axial strain of 0.10416 .

\section{Mesoscale quantities}

In the present case, meso-domains are considered in the form of grain-loops. They are defined from the tessellation of the sample area based on contacts between grains. The sample domain is seen as a unique pavement composed of grain-loops of different orders (i.e. number of grains forming the loops). High order grain-loops are on average more deformable than low order ones. This deformability is the base of microreorganizations and a way to adapt to external loadings. Non-contact or single contact grains called rattlers, often contained in high order loops, have a key role in the reorganizations and kinetic energy transfer as they can be absorbed in loop formation or be released when loops collapse.

We want to investigate the ability of mesodomains to develop kinetic energy with no external input. The second-order criterion $[3,12,13]$ is relevant for this stability analysis, provided that the continuum mechanics framework holds. According to this criterion, if a given system is at balance initially and the external second-order work is null, the system evolves from static to inertial regime if and only if the internal secondorder work is negative. At mesoscale, the continuum framework is questionable and there is no reason for the meso-domains to fulfil the requirements for this criterion to anticipate bursts of kinetic energy. However, meso-stress and meso-strain can be defined (consistently with macroscopic definitions), and a mesoscale internal second order work is formed as:

$$
\left(w_{2}\right)_{\Omega_{l}}=\langle\Delta \boldsymbol{\sigma}\rangle_{\Omega_{l}}:\langle\Delta \boldsymbol{\varepsilon}\rangle_{\Omega_{l}}
$$

with $\sigma$ a stress tensor, $\varepsilon$ a strain tensor and $\Omega_{l}$ the mesodomain of interest.

The meso-stress and meso-strain are averages of the respective quantities over $\Omega_{l}$. Using Gauss theorem, the average strain rate on the domain is defined from quantities on the boundary of the domain. Centers of neighboring spheres belonging to the same loop are 
joined by branch vectors (Fig. 3a). They form the polygon $\Omega_{l}$ on which the calculation is based. The meso-incremental strain tensor is defined as a function of a linear interpolation of the incremental displacements of the grains $[14,15]$ :

$$
<\Delta \boldsymbol{\varepsilon}>_{\Omega_{l}}=\frac{1}{\left|\Omega_{l}\right|} \sum_{k \in \delta \Omega_{l}} \frac{1}{2}\left(\Delta \boldsymbol{u}_{k}^{0}+\Delta \boldsymbol{u}_{k}^{1}\right) \otimes \boldsymbol{n}_{k} l_{k}
$$

where $\boldsymbol{u}_{k}^{0}$ and $\boldsymbol{u}_{k}{ }_{k}$ are the incremental displacement vectors of the vertices of the $\mathrm{k}^{\text {th }}$ edge, $l^{k}$ is the length of the $\mathrm{k}^{\text {th }}$ edge and $\boldsymbol{n}^{k}$ is the normal to this same edge and $\left|\Omega_{l}\right|$ is the volume of the polygon of domain $\Omega_{l}$.

The meso-stress we propose is defined as a spatial average over the polygon domain $\Omega_{l}$ of the grains micro stress (Fig. 3b). The main hypothesis is that the averaged stress calculated on the part of the grain included in the loop area is equal to the average stress of the grain. This definition is not restricted to a quasi-static regime and can be extended to dynamical regime as long as the contribution of rotations remains negligible.

$$
\langle\boldsymbol{\sigma}\rangle_{\Omega_{l}}=\frac{1}{\left|\Omega_{l}\right|} \sum_{g \in \Omega_{l}}\left|V_{g} \cap \Omega_{l}\right|\langle\boldsymbol{\sigma}\rangle_{V_{g}}
$$

Where $V_{g}$ is the solid domain of the grain.

The macroscopic axial deformation between two steps used to calculate the incremental macro stress is very small $\left(10^{-6}\right)$. Thus, we can make the following approximation:

$$
\langle\Delta \boldsymbol{\sigma}\rangle_{\Omega_{l}}=\Delta\langle\boldsymbol{\sigma}\rangle_{\Omega_{l}}
$$

(a)

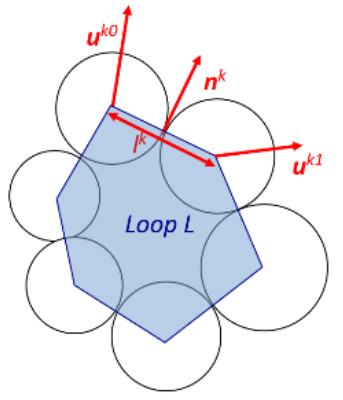

(b)

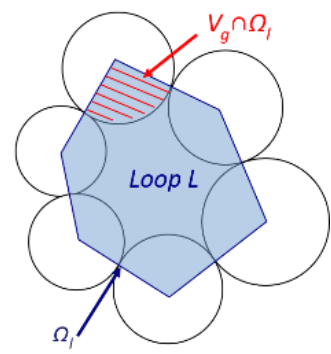

Fig. 3. Sketches to illustrate the calculation of the meso-strain (a) and meso-stress (b).

\section{Mesoscale changes during a burst of kinetic energy}

\subsection{Evolution of meso-structures during a burst of kinetic energies}

The incremental evolution of grain-loops and rattlers is followed during the burst presented in this paper inside and outside the burst zone (box shown in Fig. 1). The incremental evolution is equal to the ratio between the incremental evolution of the number of structures $\Delta N$ over a small axial strain increment $\left(10^{-6}\right)$ and their number $N_{0}$ at the beginning of the recording. Figure 4 shows the incremental evolution of the four main groups of grain-loops inside the burst area (Fig. 4a) and outside (Fig. 4b). A small and equal amount of loops are lost and created for each type of loops. This observation is consistent with the fact that the loop decomposition of the microstructure is statistically at equilibrium at critical state. The burst does not induce noticeable statistical changes on the loop population. However, we can notice that low order loops are broken first (peak of lost loops before the peak of energy) and created after (peak of gain loops just after the peak of kinetic energy). For the $6+$ order loops, the maximum of created and lost incremental evolutions occurs simultaneously. The incremental evolution is also drawn for the rattlers and it shows that rattlers are first created and then lost, which can be related to the evolution of grain-loops. Before the maximum of kinetic energy, a significant proportion of loops of order 3, 4 and 5 are destroyed, transformed into higher order loops and creates rattlers. After the peak, these same orders of loops are reformed back and thus consume rattlers. Meanwhile, the high order loops are constantly created and lost and ensure statistical stability (in terms of loop composition) of the zone concerned by an inertial transition. By contrast, outside the burst zone, no significant changes are spotted neither for the grainloops (Fig. 4b) nor the rattlers. The changes due to the burst of kinetic energy remains in the neighborhood of its creation and immediate propagation.

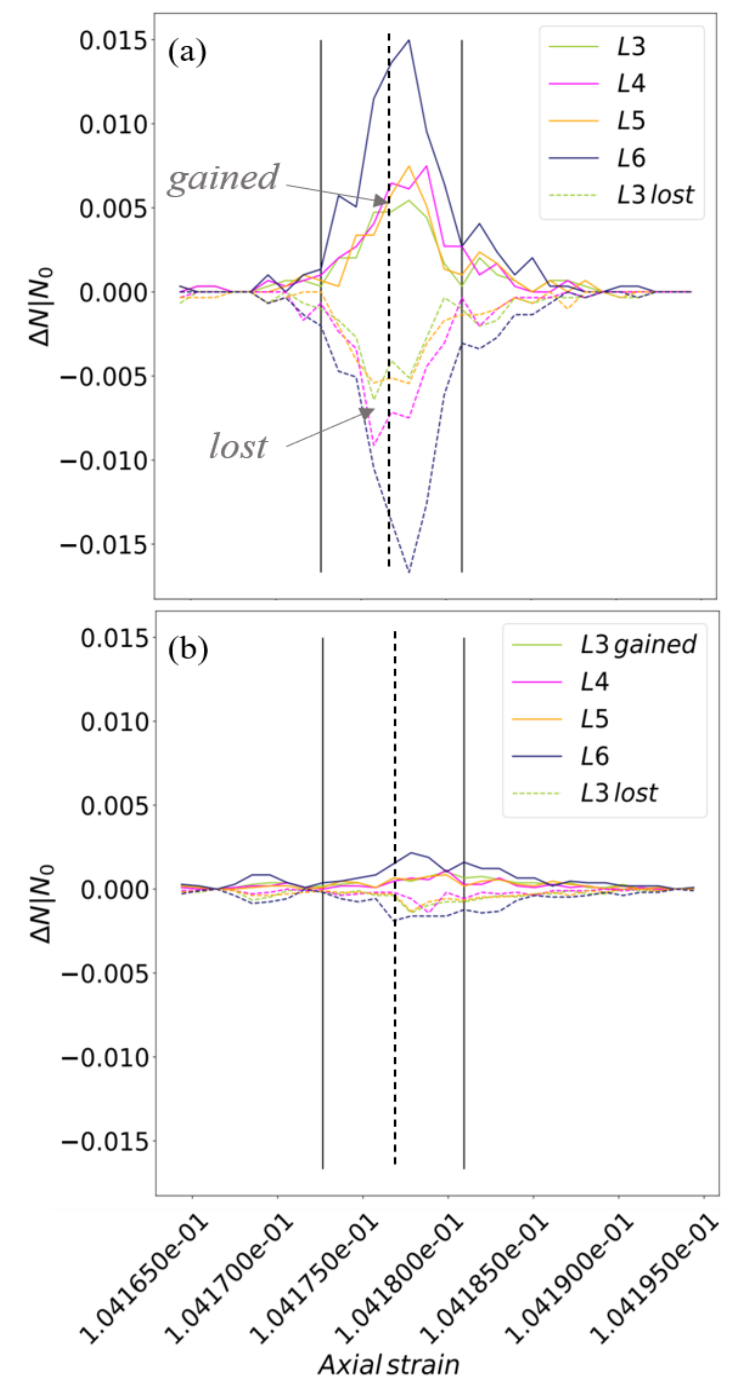

Fig. 4. Incremental evolution of grain-loops inside (a) and outside (b) the burst domain shown in Figure 1. A color is assigned to each order loops (L3, L4, L5 and L6+). Solid lines represent the creation of loops $(\Delta N>0)$ and dotted lines represents the loss of loops $(\Delta N<0)$. The duration of the burst is delimited by the vertical solid lines and the peak of maximum kinetic energy is displayed by the vertical dotted line. 


\subsection{Evolution of mesoscopic second order work during a burst of kinetic energy}

In Fig. 5, the spatial distribution of the mesoscale second-order work (Eq. (2)) for macroscopic increments of axial strain of $10^{-6}$ shows that the vanishing of the mesoscopic second order work follows the spatial evolution of the kinetic energy (Fig. 5) in the triggering mechanisms. It should be noticed that the burst area is not formed only from loops with negative values of second-order work. Positive and negative values follow the burst displacements, while the rest of the sample shows values close to zero. However, by looking at the volume-averaged second-order work in the burst domain, we can exhibit the predominance of negative values before the onset of the burst. The vanishing of the average second-order work based on meso-stress and meso-strain definitions can be seen as a signature of instability in the domain, while the followed increase indicates that the microstructure reorganizations stabilize the area and inhibit the growth of the burst.

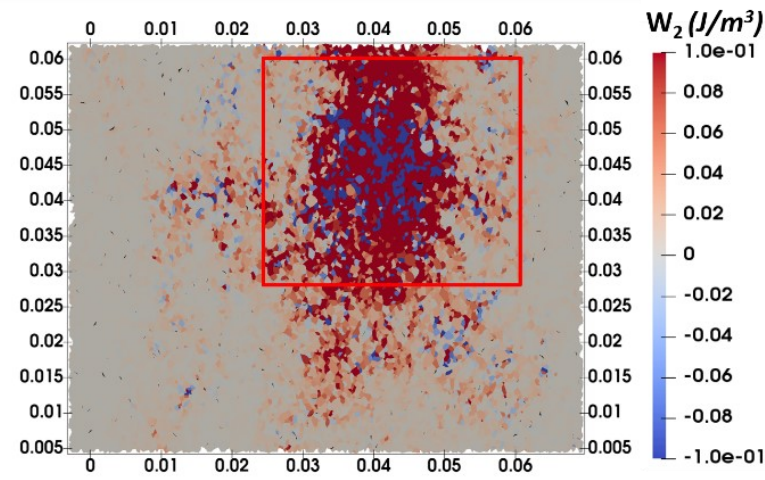

Fig. 5. Screenshot of the presented burst. The meso domains are colored in function of their internal second-order work. The box used for the delimitation of the burst is superimposed to the screenshot. We notice that the extension of the variations of the meso internal second order work is wider than the micro kinetic energy and highlight zones will be soon concerned by the burst of kinetic energy.

\section{Conclusion and outlooks}

We have shown the relevance of defining meso-domains as grain loops in granular material to analyze bursts of kinetic energy. Modifications in the number and of the nature of grain loops is synonym of microstructure reorganizations and release in kinetic energy. The microstructure after the burst is very similar to the one before, underlining the statistical equilibrium at critical state. Moreover, precursors of inertial transition have been pointed out in the zone of the burst of kinetic energy. A large number of contacts close to sliding in an area is necessary to trigger the kinetic energy release. The variations in the second-order work, defined at mesoscopic scale, anticipate the burst of kinetic energy and give detailed information on its spatial origin. The influence of the material density (from loose to dense) will be studied to confirm and generalize the presented results. Mesoscopic quantities introduced in this work are based on several assumptions that need to be clarified and investigated in order to validate the interpretation of meso-second order work from a physical point of view. In particular, the contribution of inertial effects should be assessed.

\section{References}

[1] B. Cambou, M. Jean, F. Radjaï, (Eds.): Micromechanics of granular materials. John Wiley \& Sons (2013)

[2] D Vescovi, D. Berzi, C. di Prisco, Granular Matter, 20(2), 27 (2018)

[3] F. Darve, G. Servant, F. Laouafa, H. D. V. Khoa, Computer methods in applied mechanics and engineering, 193(27-29), 3057-3085 (2004)

[4] A. Wautier, S. Bonelli, F. Nicot, Int. J. Numer. Anal. Methods Geomech.,42(9), 1037-1056 (2018a)

[5] J. Gaume, T. Gast, J. Teran, A. van Herwijnen, C. Jiang, Nature Communications, 9(1), 1-10 (2018)

[6] D.M. Walker, A. Tordesillas, G. Froyland, Physical Review E, 89(3), 032205 (2014)

[7] J Liu, F. Nicot, W. Zhou, Powder Technology, 338, 458-470 (2018)

[8] H. Zhu, F. Nicot, F. Darve, Granul. Matter, 18(1), 3 (2016)

[9] V. Šmilauer, E. Catalano, B. Chareyre, et al. Yade documentation, 2010.

[10] P.A. Cundall, O.D. Strack, Geotechnique, 29(1), 47-65 (1979)

[11] A. Clerc, A. Wautier, S. Bonelli, F. Nicot. Mesoscale signatures of inertial transitions in granular materials. Granular Matter (2021) accepted

[12] F. Nicot, F. Darve, Int. J. Solids Struct., 44(20), 6630-6652 (2007)

[13] R. Wan, F. Nicot, F. Darve, ISTE Press - Elsevier (2017)

[14] S. Bonelli, S., O. Millet, F. Nicot, D. Rahmoun, De Saxcé G, Int. J. Solids Struct., 49, 947-958 (2012)

[15] N.S. Nguyen, H. Magoariec, B. Cambou, B, Int. J. Numer. Anal. Methods Geomech., 36(14), 16091635 (2012) 